\title{
NOTICIARIO
}

\section{LA PALEOPALINOLOGÍA EN EL LABORATORIO DE ARQUEOBOTÁNICA DEL DEPARTAMENTO DE PREHISTORIA, INSTITUTO DE HISTORIA, CSIC: 1977-2002}

\author{
PALEOPALYNOLOGY AT THE LABORATORY OF ARCHAEOBOTANY OF THE \\ DEPARTMENT OF PREHISTORY, INSTITUTE OF HISTORY, CSIC: 1977-2002
}

\author{
PILAR LÓPEZ GARCÍA $(*)$ \\ JOSÉ ANTONIO LÓPEZ SÁEZ (*) \\ ROSARIO MACÍAS ROSADO $(*)$
}

\begin{abstract}
RESUMEN
Con motivo de los 25 años de la puesta en marcha del Laboratorio de Arqueobotánica del Departamento de Prehistoria (Instituto de Historia, CSIC) se presentan los resultados de su actividad a lo largo de este periodo de tiempo: proyectos, líneas de investigación abordadas y bibliografía generada en su seno.
\end{abstract}

\begin{abstract}
Taking the opportunity to commemorate the 25th anniversary of the Laboratory of Archaeobotany of the Department of Prehistory (Institute of History, CSIC), this paper presents its activity through this period: projects, research themes and the literature published by its researchers.
\end{abstract}

Palabras clave: Laboratorio Arqueobotánica. Departamento Prehistoria. Ciencia en Arqueología.

Key words: Laboratory of Archaeobotany, Department of Prehisitory. Science in Archaeology.

\section{INTRODUCCIÓN}

La tradición paleopalinológica española iniciada en los años 40 por Bellot y Vieitez se había centra-

(*) Laboratorio de Arqueobotánica, Dpto. de Prehistoria. Instituto de Historia. CSIC. Duque de Medinaceli 8. 28014 Madrid. Correo electónico: plopez@ceh.csic.es alopez@ceh.csic.es rmacias@ceh.csic.es

Recibido: 20-XI-2001; aceptado: 27-I-2002. do, entre otras, ya en la década de los 50, en las figuras de los Dres. Menéndez-Amor y Florschuzt. Botánicos ambos, habían realizado un magnífico trabajo en el campo de la paleobotánica sobre depósitos naturales, y en algún caso habían abordado el análisis de algunos depósitos arqueológicos. Dejando aparte los laboratorios que han seguido una línea estríctamente botánica centrada en depósitos naturales, señalaremos que los laboratorios dedicados, entre otros, a depósitos arqueológicos son escasos. Se trata, en muchos casos, de grupos que abordan el estudio de yacimientos localizados dentro de sus límites regionales de trabajo.

El propósito de este trabajo es presentar el Laboratorio de Arqueobotánica del Departamento de Prehistoria del Instituto de Historia del CSIC. El hecho de hacerlo ahora se justifica por cumplirse 25 años de su creación. A pesar de su nombre, vamos a centrarnos en el estudio de una de las tres líneas en las que está dividido, la Palinología. La razón de ello se debe a que es precisamente ésta la que lleva desarrollando su actividad todo este tiempo y por ser la que verdaderamente está consolidada, no tanto por elección propia, como por las circunstancias laborales que no han facilitado que las otras dos, Carpología y Antracología estén cubiertas con personal fijo.

El Laboratorio se crea a finales de 1977, gracias al apoyo del Profesor M. Almagro Basch, que obtiene fondos suficientes para montar los primeros equipos en la que sería su primera sede, el Instituto Español de Prehistoria, actual Dpto. de Prehistoria, en el Museo Arqueológico Nacional. Uno de nosotros (P. López) inicia la investigación palino- 
lógica en el ámbito de la Arqueología, desplazándose durante los años 1976-77 al Laboratorio de Palinología de Mme. Arl. Leroi-Gourhan en el Museo del Hombre de París. Desde el primer momento, y de acuerdo con el proyecto inicial, la investigación se centró en sedimentos procedentes de contextos arqueológicos españoles, ya que éstos eran tradicionalmente analizados en laboratorios franceses, no habiendo ninguno español dedicado específicamente a ellos.

El laboratorio comienza sus trabajos en 1978 contando con el apoyo de Nieves Cajal realizando los primeros análisis químicos. P. López García (1978) publica una síntesis sobre el Holoceno de la Península Ibérica, un hito importante en la sociedad arqueo-palinológica española, por ser un trabajo en el que se aglutinaban todos los datos de España y Portugal, tanto referidos a depósitos naturales como arqueológicos. Más tarde, la misma autora y colaboradores abordan nuevas síntesis, tanto nacionales como regionales (López García, 1985a, 1986b, 1987a, 1988a, 1992a, b, 1997, 2000; López García y López Sáez, 1994a, b; López Sáez y López García, 1994, 1999; Macías Rosado et al., 1998), que aclararon la panorámica paleopalinológica ibérica. Al mismo tiempo inician estudios arqueobotánicos en zonas carentes de ellos como era el caso de ambas Mesetas, Andalucía o Extremadura.

Paralelamente López García (1981d, 1982e, 1986a, 1989a a, b, 1994), López García et al. (1997a, b) y López Sáez y López García (1992) han ido poniendo de manifiesto la importancia de la paleopalinología en ámbitos arqueológicos, resaltando su enorme valor en varios aspectos como la interpretación paleoecológica, la dinámica de las poblaciones humanas respecto a la evolución de su hábitat, la definición de sus estrategias adaptativas, o la cronología y periodización de la introducción de los cultivos. Incluso López García (1984b, 1986a, 1989a, b) ha propuesto algunas alternativas al tratamiento químico habitual de las muestras palinológicas procedentes de contextos arqueológicos.

A pesar de lo expuesto, la investigación llevada a cabo en el Laboratorio ha pasado ocasionalmente desapercibida en alguna de las publicaciones recientes. Se trata de estudios polínicos de carácter general, incluyendo los paleopalinológicos (Gutiérrez Bustillo, 1994; Sáenz Laín y Gutiérrez Bustillo, 1991) o centrados exclusivamente en la aplicación de éstos a la reconstrucción paleoclimática (Salas, 1995). Este mismo hecho se ha constatado en la reciente revisión palinológica del Holoceno Ibérico (Martínez Atienza, 1999), periodo paleoclimático objeto de la gran mayoría de los trabajos de nuestro laboratorio. Para paliar este hecho y reconociendo la dificultad, en ciertas ocasiones de acceder a toda la bibliografía paleopalinológica publicada, hemos creado una página web en la que se irá actualizando la información y cuya dirección es http://www.prehistoria.ceh.csic.es/prehistoria/ amed/index.html

Con la presentación de este trabajo queremos lograr dos objetivos:

1) Reunir las publicaciones del Laboratorio de Arqueobotánica del CSIC en Madrid.

2) Difundir nuestras actividades en el seno de la comunidad científica nacional e internacional en el campo de la paleopalinología, principalmente de la arqueopalinología.

El Laboratorio cuenta en la actualidad con una buena infraestructura científica, tanto en lo que se refiere al equipamiento que permite la realización de análisis químicos, como a la bibliografía, contando con un número importante de revistas científicas específicas. Cuenta además con una magnífica colección de referencia.

Ha sido base de formación de palinólogos españoles que trabajan, tanto en nuestro Centro como en otros nacionales o extranjeros. En su seno se han elaborado Tesis de licenciatura y Tesis doctorales. Se han impartido cursos de Doctorado en varias Universidades españolas, Seminarios en distintas Instituciones y se han establecido Acciones integradas y distintos modos de cooperación científica con algunos laboratorios internacionales. Sus miembros han realizado estancias de especialización en Laboratorios europeos y americanos (Alemania, Francia, Holanda, Inglaterra, Méjico)

\section{PROYECTOS DE INVESTIGACIÓN}

Los Proyectos están ordenados por su fecha de realización y, salvo indicación, la investigadora principal de los mismos ha sido P. López.

-1986-1989

ID-831 Aspectos socioeconómicos y culturales del inicio de la metalurgia en el SE español: un modelo para la definición del cambio cultural. CAICYT.

$-1988-1990$

Paleoecología y prehistoria de un paisaje rural. Estudio de la Sierra de O Bocelo (A Coruña). In-

T. P., 59, n. ${ }^{\circ} 1,2002$ 
vestigador Principal: Felipe Criado Boado. Xunta de Galicia.

$-1990-1992$

PB88-080 La dialéctica hombre medio en el mediodía peninsular: la perspectiva arqueológica». DGICYT.

$-1991-1994$

Mapa de paleovegetación de la Comunidad de Madrid. Consejería de Cultura de la Comunidad de Madrid.

-1993-1996

PB92-0073 Elementos antrópicos en los diagramas polínicos españoles: la Meseta como modelo. DGICYT.

$-1993-1996$

La transición del Paleolítico medio al superior en la cornisa cantábrica, aspectos culturales, biológicos y geocronológicos. DGICYT. Investigadora principal Victoria Cabrera Valdés.

-1996-1999

PS95-0031 El inicio de la economía productiva en la gran estepa euroasiática y su impacto en el medio-ambiente: ¿catástrofes ecológicas en la estepa? DGICYT. Investigadora principal $\mathrm{M}^{\mathrm{a}}$ IsabeI Martínez Navarrete.

-1999- 2001

PB97-1126 Los sedimentos arqueológicos de la Edad del Bronce de la Bahía de Cádiz, base de un estudio arqueobotánico. DGES.

$-1999-2000$

06/0092/98 El paisaje holoceno de Madrid a través del estudio arqueobotánico de yacimientos situados en el Oeste de la Comunidad. Comunidad de Madrid.

$-1999-2002$

PR269/98-8196 Territorio Nurágico y paisaje antiguo del Altiplano de Praemuru: un Proyecto / de investigación y patrimonialización en el distrito de Orroli (Nuoro, Cerdeña). Universidad Complutense de Madrid. Investigadora principal $\mathrm{M}^{\mathrm{a}}$ Luisa Ruíz-Galvez.

$-2000-2001$

IFD97-1529 Evolución humana y del paisaje en las comarcas de la Serena y la Vera: dos modelos para entender el desarrollo histórico de Extremadura. Plan Nacional I+D dentro del programa FEDER.

$-1999-2002$

PB98-0653 Investigación arqueometalúrgica y arqueobotánica para la evaluación de la metalurgia del cobre en Kargaly (Urales del Sur, Región de Orenburg, República Federativa Rusa). DGES.
Investigadora principal $\mathrm{M}^{\mathrm{a}}$ Isabel Martínez-Navarrete.

$-2001-2004$

BHA 2001-2308 Estudio arqueológico y paleoambiental de la Comarca Natural de la Serena: una vía para potenciar la economía y el turismo sostenido en Extremadura. DGES.

\section{LÍNEAS DE INVESTIGACIÓN Y RESULTADOS}

Tenemos que señalar que las líneas que a continuación se presentan responden a diferentes objetivos que nos hemos ido marcando a lo largo de estos años. En unas ocasiones han sido resultado de propuestas propias para estudiar una determinada región o momento cultural y en otras han sido respuesta a peticiones de colaboración de otros equipos. En cualquiera de los casos el objetivo final, antes y ahora, es abordar, en la medida de lo posible, estudios de carácter regional o, en su defecto, análisis de depósitos que conformen un conjunto capaz de darnos características generales del área estudiada.

a) El medio natural en la transición Epipaleolítico-Neolítico en el Valle Medio del Ebro.

Referencias: López García (1987a, b; 1992a , b), López García et al. (1991) y López García y López Sáez (2000b)

b) Problemática biogeográfica y naturalidad de las formaciones de Pinus nigra, Castanea y Betula en el sur de la Sierra de Gredos.

Referencias: López Sáez (1992, 1993, 1995a, b), López Sáez y López García (1994) y López Sáez et al. (1991, 1996, 1997, 1999).

c) Antropización durante el Holoceno medio y reciente en Galicia. La problemática de la introducción del cereal.

Referencias: Aira et al. (1992), López García (1982d, 1984c, d), López García et al. $(1992,1993)$ y López García y López Sáez (1993).

d) La vegetación prehistórica y protohistórica en Andalucía; la problemática arqueobotánica de la presencia de Olea y Vitis; la neolitización regional y la introducción del cereal; aspectos paleofitogeográficos y diferencias tafonómicas entre depósitos arqueológicos y naturales.

Referencias: López García (1982a, 1984e, 1985a, 1988a, b), López García y López Sáez (1994a, b, d, 1996d, 1997, 1998).

e) La transición Tardiglaciar-Holoceno en el Levante peninsular y la problemática del Dryas II. 
Referencias: Cacho et al. (1995) y López Sáez y López García (1999).

f) Paleovegetación y Antropización del Paleolítico Superior al Mesolítico en la Cordillera Cantábrica.

Referencias: López García (1981a, b) y López García et al. (1996a).

g) La paleovegetación de la Comunidad Autónoma de Madrid y la dinámica de la antropización.

Referencias: López García (1983c, 1997), López García y Arnanz (1994) y Macías Rosado et al. (1996).

h) El valor paleoecológico de los microfósiles no polínicos en Palinología Arqueológica.

Referencias: López Sáez et al. (1991, 1998a).

i) Relación entre la investigación palinológica y las posibles catástrofes ecológicas causadas por actividades minero-metalúrgicas, en la Gran Estepa Euroasiática (Rusia).

Referencias: López García et al. (1996b), Vicent et al. (2000) y López et al. (2001)

j) Evolución de la vegetación en el Norte y Sur de la región extremeña

La tabla I refleja todos los muestreos y análisis en los que han participado miembros del Laboratorio de Arqueobotánica, tanto en depósitos naturales como arqueológicos. Una síntesis de todos estos datos, así como las principales problemáticas de nuestra investigación fue presentada en el último First CiMBIO (CircumMediterranean BIOmes) Workshop, celebrado en Jena (Alemania) en 2000. Cada depósito se identifica por su nombre, ubicación regional dentro y fuera de la Península Ibérica, su asignación cultural -salvo los depósitos naturales-, periodos paleoclimáticos que comprende $y$, finalmente, la bibliografía. Los inéditos corresponden a estudios en prensa, evaluación o a muestras estériles polínicamente. En cambio, los "en curso" corresponden a aquellos cuyas muestras están sin procesar o pendientes de análisis palinológico.

\section{AGRADECIMIENTOS}

Ya que es la primera vez que hacemos un estudio recopilatorio de lo que ha acaecido en estos años en el Laboratorio, queremos dejar patente el agradecimiento a aquellas personas que hicieron posible que éste fuera una realidad. En primer lugar el Prof. Almagro Basch, que entendió la importancia de este tipo de investigación y facilitó la estancia de uno de nosotros (P.L.) en París en el final de los 70, fecha en la que realizar una estancia en el extranjero no era tan habitual como lo es hoy. Seguramente en este repaso, alguien quedará sin nombrar, a pesar de haber pasado a formar parte de los recuerdos más agradables de aquellos años. Intentaremos que sean los menos posibles. Recuerdo especial dedicamos al Dr. A. Leroi-Gourhan y todo su equipo por acoger a Pilar López en su Laboratorio como uno mas de ellos a pesar de ser una stagiaire del laboratorio contiguo al suyo. El mas firme de los reconocimientos a Mme. Arlette Leroi-Gourhan, alma del Laboratorio de Palinología del Museo del Hombre en aquellos años. Su cariño y ayuda contribuyeron a que su formación llegara a ser la que hoy es. Gracias a Dhin Trong-Hieu y Monique Olive por ensenar el pesado proceso químico y hacerle pasar los mejores años de su vida predoctoral. Un recuerdo imborrable a Michel Girard al que volvería a acudir años más tarde en el Laboratorio de Palinología del CRA en Valbonne. Su sentido del humor e inmensa sabiduría botánica y polínica hicieron las cosas mucho más fáciles. Finalmente gracias a todos los colegas con los que ha tenido la oportunidad de trabajar posteriormente, y ya desde una posición laboral estable.

No queremos olvidar a las personas que, en un momento dado, formaron parte del Laboratorio de Arqueobotánica del CSIC (Madrid) y contribuyeron al desarrollo de sus investigaciones paleopalinológicas. Algunas son coautoras de varios de los artículos citados. Ellas son, por orden alfabético, Nieves Cajal, Pedro Díaz, Justo Díez, Paula Gil, Ana Hernández, María José Istúriz, Roberto Jiménez, Mariano Martín, autor del primer diseño de nuestra página Web y coautor en algunas de nuestras publicaciones, Almudena Orejas, Paloma Rodríguez, Aurora Ruíz, María Fernanda SánchezGoñi, Juan José Sánchez-Villapadierna e Isaac Vega.

Nuestro agradecimiento debe llegar también a Dña. Ana M. Arnanz y la Dra. Paloma Uzquiano, quienes desarrollan las otras dos líneas de investigación fundamentales en el Laboratorio de Arqueobotánica, la Carpología y la Antracología respectivamente.

Especial mención merece R. Macías (co-autora), responsable del procesado de la mayoría de las muestras en el Laboratorio desde su creación e incorporada en los últimos años a la identificación microscópica. 
Tab. I. Relación de análisis palinológicos llevados a cabo en el Laboratorio de Arqueobotánica del CSIC (Madrid), periodización cultural y cronológica (PERIODO CULTURAL COMPRENDIDO: PI: Paleolítico Inferior; PM: Paleolítico Medio; PS: Paleolítico Superior; M: Mesolítico; N: Neolítico; C: Calcolítico; B: Edad del Bronce; PR: Periodo Prerromano; R: Periodo Romano; EM: Edad Media; EC: Edad Contemporánea; PERIODO PALEOCLIMAÁTICO COMPRENDIDO: PG: anterior al Tardiglaciar; OD: Dryas antiguo; B/A: Bölling/Allërod; YD: Dryas reciente; PB: Preboreal; BO: Boreal; A: Atlántico; SB: Subboreal; SA: Subatlántico; PR: reciente, lluvia polínica.)

\begin{tabular}{|c|c|c|c|c|c|c|c|c|c|c|c|c|c|c|c|c|c|c|c|c|}
\hline Yacimiento & \begin{tabular}{|l|} 
Provincia/Departamen. \\
\end{tabular} & \begin{tabular}{|l|l|l|} 
PI & PI \\
\end{tabular} & \begin{tabular}{l|l}
$\mathrm{M}$ & $\mathrm{PS}$ \\
\end{tabular} & $M$ & $\mathrm{~N}$ & $\mathrm{C}$ & \begin{tabular}{l|l} 
B & 1 \\
\end{tabular} & 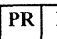 & \begin{tabular}{l|l}
$\mathbf{R}$ & $\mathrm{E}$ \\
\end{tabular} & \begin{tabular}{l|l} 
EM & EC \\
\end{tabular} & c) $\mathbf{P G}$ & OD & $\mathrm{B} / \mathrm{A}$ & YY & PB & BO & \begin{tabular}{|ll}
$\mathbf{A}$ \\
\end{tabular} & SB & $\mathrm{SA}$ & \begin{tabular}{l|l|} 
PR & Referencia \\
\end{tabular} \\
\hline PETÓN & A Coruña & & & & $\mathrm{x}$ & & & & & & & & & & & & $\mathrm{x}$ & & & $\begin{array}{ll} & \text { López García et al., } 1992 \\
\end{array}$ \\
\hline MAZARELAS & A Coruña & & & & $\mathrm{x}$ & & & & & & & & & & & & $\mathrm{x}$ & $\mathrm{x}$ & & López García et al.. 1992 \\
\hline \begin{tabular}{|l} 
FORNO VARELA \\
\end{tabular} & A Coruña & & & & $\mathrm{x}$ & & & & & & & & & & & & & $\mathrm{x}$ & $\mathrm{x}$ & López García et al., 1993 \\
\hline CRUZ DO BOCELO & A Coruña & & & & $\mathrm{x}$ & & & & & & & & & & & & & $\mathrm{x}$ & $\mathrm{x}$ & López García et al., 1993 \\
\hline TORRE DOS MOUROS & A Coruña & & & & $\mathrm{x}$ & & & & & & & & & & & & & $\mathrm{x}$ & $x$ & López García et al., 1993 \\
\hline FORNO DOS MOUROS & A Coruña & & & & $\mathrm{x}$ & & & & & & & & & & & & & $\mathrm{x}$ & $\mathrm{x}$ & López Garcia et al., 1993 \\
\hline MORCIGUEIRA & A Coruña & & & & $\mathrm{x}$ & & & & & & & & & & & & & $\mathrm{x}$ & $\mathrm{x}$ & López Garcia et al.,1993 \\
\hline AMENEIROS & A Coruña & & & & & & & & & & & & & & & & $\mathrm{x}$ & $\mathrm{x}$ & $\mathrm{x}$ & Aira et al., 1992 \\
\hline A PENA & A Coruña & & & & & & & & & & & & & & & & & 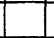 & $\mathrm{x}$ & Aira et al., 1992 \\
\hline \begin{tabular}{|l} 
CHAN DA CRUZ \\
\end{tabular} & A Coruña & & & & & & & & & & & & & & & & & 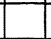 & $\mathrm{x}$ & Aira et al., 1992 \\
\hline INSUA & A Coruña & & & & & & & & & & & & & & & & $x$ & $\mathrm{x}$ & $x$ & Aira et al., 1992 \\
\hline LAGOA & A Coruña & & & & & & & & & & & & & & & & & $x$ & $\mathrm{x}$ & Aira et al., 1992 \\
\hline MUIÑO & A Coruña & & & & & & & & & & & & & & & & . & $x$ & $x$ & Aira et al., 1992 \\
\hline ARENAZA & Alava & & & & $\mathrm{x}$ & & & & & & & & & & & & $\mathrm{x}$ & & 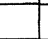 & Estéril \\
\hline NECROPOLIS DE LOS VILLARES & Albacete & & & & & & & $\mathrm{x}$ & & & & & & & & & & & $\mathrm{x}$ & En prensa \\
\hline TOSSAL DE LA ROCA & Alicante & & $\mathrm{x}$ & & & & & & & & & $x$ & $\mathrm{x}$ & & & $\mathrm{x}$ & $x$ & & & $\begin{array}{l}\text { Cacho et al., 1983, 1995, 1998a, b; López Garcia, 1986b; López } \\
\text { Sáez y López Garcia, 1999 }\end{array}$ \\
\hline EL BARRANC & Alicante & & $\mathrm{x}$ & & & & & & & & $x$ & & & & & & & & & Estéril \\
\hline CERRO VIRTUD & Almería & & & & $\mathrm{x}$ & & & & & & & & & & & & $\mathrm{x}$ & & & Estéril \\
\hline CUEVA AMBROSIO & Almería & & $x$ & & & & & & & & & & & & $x$ & & & & & López García, 1988a, b; López García y López Sáez, 1994a, b \\
\hline ALMIRAZAQUE & Almería & & & & & $\mathrm{x}$ & & & & & & & & & & & & $\mathrm{x}$ & & $\begin{array}{l}\text { López García, 1986b, 1988a, 1994; López García y López Sáez, } \\
\text { 1994a, b }\end{array}$ \\
\hline CORE ALBORAN 11-P & Almería & & & & & & & & & & $\mathrm{x}$ & $\mathrm{x}$ & $\mathrm{x}$ & $\mathrm{x}$ & $\mathrm{x}$ & $\mathrm{x}$ & $\mathrm{x}$ & $\mathrm{x}$ & $\mathrm{x}$ & López Sáez y López García, 1999; Targarona et al.,1996 \\
\hline NICE AEROPORT & Alpes Maritimes, Francia & & & & & & & & & & & & & $x$ & $x$ & $x^{-}$ & $x$ & $x$ & $\mathrm{x}$ & López Sáez et al., 1998a \\
\hline GARIBALDI & \begin{tabular}{|l|} 
Alpes Maritimes, Francia \\
\end{tabular} & & & & & & & & & & & & & & & & $\mathrm{x}$ & $\mathrm{x}$ & $\mathrm{x}$ & En prensa \\
\hline VALLEE DES MERVEILLES & Alpes Maritimes, Francia & & & & & & & & & & & $\mathrm{x}$ & $\mathrm{x}$ & $\mathrm{x}$ & $\mathrm{x}$ & $\mathrm{x}$ & $\mathrm{x}$ & $\mathrm{x}$ & $\mathrm{x}$ & Inédito \\
\hline PENDIMOUN & Alpes Maritimes, Francia & & & & $\mathrm{x}$ & & & & & & & & & & & & $\mathrm{x}$ & & & En prensa \\
\hline CAUCADE & Alpes Maritimes, Francia & & & & $\mathrm{x}$ & & & & & & & & & & & & $\mathrm{x}$ & & & En prensa \\
\hline SAINT ROCH & Alpes Maritimes, Francia & & & & & & & & & & & & & & & & $x$ & & & En prensa \\
\hline $\begin{array}{l}\text { PALAIS DES EXPOSITIONS DE } \\
\text { NICE }\end{array}$ & Alpes Maritimes, Francia & & & & & & & & & & & & & & & & $\mathrm{x}$ & & & En prensa \\
\hline PALAIS DE JUSTICE DE NICE & Alpes Maritimes, Francia & & & & & & & & & & & & & & & & $\mathrm{x}$ & & & En prensa \\
\hline LOS AZULES & Asturias & & & $x$ & & & & & & & & & & & $\mathrm{x}$ & & & 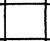 & 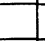 & López García, 1981b, 1986 \\
\hline CUEVA DEL BUXU & Asturias & & $\mathrm{x}$ & & & & & & & & $\mathrm{x}$ & & & & & & & & & Inédito \\
\hline MAZACULOS & Asturias & & & $\mathrm{x}$ & & & & & & & & & & & $\mathrm{x}$ & $\mathrm{x}$ & & & & López García, 1986b \\
\hline SAN ESTEBAN DEL VALLE & Avila & & & & & & & & & & & & & & & & & $\mathrm{x}$ & $\mathrm{x}$ & López Sáez et al., 1997 \\
\hline EL RASO DE CANDELEDA & Avila & & & & & & & $\mathrm{x}$ & & & & & & & & & & & $\mathrm{x}$ & $\begin{array}{l}\text { López García, 1984a, 1985a, 1986b, 1994; López Sáez y López } \\
\text { García, 1994; López Sáez et al., 199 }\end{array}$ \\
\hline PUERTO DE CASILLAS & Avila & & & & & & & & & & & & & & & & & & $\mathrm{x}$ & López Sáez et al., 1996, 1998a \\
\hline \begin{tabular}{|l} 
GARGANTA DEL VILLAR \\
\end{tabular} & Avila & & & & & & & & & & & & & & & & $\mathrm{x}$ & $\mathrm{x}$ & $\mathrm{x}$ & Inédito \\
\hline
\end{tabular}




\begin{tabular}{|c|c|c|c|c|c|c|c|c|c|c|c|c|c|c|c|c|c|c|c|c|c|}
\hline Yacimiento & Provincia/Departamen. & $\mathrm{PI}$ & \begin{tabular}{|l|l|l}
$\mathrm{PM}$ & $\mathrm{F}$ \\
\end{tabular} & PS & M & $\mathrm{N}$ & $\begin{array}{ll}\mathrm{C} \\
\end{array}$ & \begin{tabular}{|l|l|} 
B \\
\end{tabular} & \begin{tabular}{|l|l|}
$\mathrm{PR}$ & \\
\end{tabular} & \begin{tabular}{|l|l}
$\mathbf{R}$ & $\mathbf{B}$ \\
\end{tabular} & \begin{tabular}{|l|l|l|} 
EM & E \\
\end{tabular} & \begin{tabular}{|l|l}
$E C$ & $P$ \\
\end{tabular} & \begin{tabular}{|l|l|l|}
$P G$ & 01 \\
\end{tabular} & \begin{tabular}{|l|l|}
$\mathrm{D}$ & $\mathrm{B} / \mathrm{A}$ \\
\end{tabular} & \begin{tabular}{l|l}
$\mathrm{A}$ & $\mathrm{YI}$ \\
\end{tabular} & \begin{tabular}{|l|l|}
$\mathrm{D}$ & $\mathrm{PB}$ \\
\end{tabular} & BO & $\mathrm{A}$ & $\mathrm{SB}$ & \begin{tabular}{|l|l|l}
$S A$ & $P$ \\
\end{tabular} & \begin{tabular}{l|l|} 
PR & Referencia \\
\end{tabular} \\
\hline LANZAHITA & Avila & & & & & & & & & & & & & & & & & & $?$ & $\mathrm{x}$ & \begin{tabular}{|l|l|} 
& López Sáez et al., 1999 \\
\end{tabular} \\
\hline LA TETA & Avila & & & & & & & & $\mathrm{x}$ & & & & & & & & & & & $x$ & Inédito \\
\hline EL PICUEZO & Avila & & & & & & & & $x$ & & & & & & & & & & & $x$ & Inédito \\
\hline CUESTA DEL CABALLERO & Avila & & & & & & & & $x$ & & & & & & & & & & & $x$ & Inédito \\
\hline EL TOMILLAR & Avila & & & & & & & & $\mathrm{x}$ & & & & & & & & & & & $x$ & Inédito \\
\hline LOS ITUEROS & Avila & & & & & & & & $\mathrm{x}$ & & & & & & & & & & & $x$ & Estéril \\
\hline ALDEAGORDILLO & Avila & & & & & & & & $\mathrm{x}$ & & & & & & & & & & & $x$ & Estéril \\
\hline VALDEPRADOS & Avila & & & & & & & & $x$ & & & & & & & & & & & $x$ & Estéril \\
\hline \begin{tabular}{|l|} 
GRAVERA DE PUENTE VIEJO \\
\end{tabular} & Avila & & & & & & & & $\mathrm{x}$ & & & & & & & & & & & $\mathrm{x}$ & Estéril \\
\hline \begin{tabular}{|l|} 
CASTREJON DE CAPOTE \\
\end{tabular} & Badajoz & & & & & & & & $x$ & & & & & & & & & & . & $x$ & En curso \\
\hline LA PIJOTILLA & Badajoz & & & & & & $\mathrm{x}$ & $x$ & & & & & & & & & & & $\mathrm{x}$ & . & Estéril \\
\hline BASKIRIA TERRAZA & Baskiria, Rusia & & & & & & & & & & & & & & & & & & & $x$ & Vicent et al., 2000 \\
\hline BASKIRIA FABRICA & Baskiria, Rusia & & & & & & & & & & & $x$ & & & & & & & . & $x$ & Vicent et al., 2000 \\
\hline CANEDOTES & Beira Alta, Portugal & & & & & & & $\mathrm{x}$ & & & & & & & & & & $\mathrm{x}$ & $\mathrm{x}$ & $x$ & En prensa \\
\hline \begin{tabular}{|l} 
CRYPTOPORTIQUES D'ARLES \\
\end{tabular} & $\begin{array}{c}\text { Bouches du Rhône, } \\
\text { Francia }\end{array}$ & & & & & & & & & $x$ & & & & & & & & & & $\mathrm{x}$ & En prensa \\
\hline PICO ROMERO & Burgos & & & & & & & $\mathrm{x}$ & & & & & & & & & & & $\mathrm{x}$ & & En prensa \\
\hline VILLASVIEJAS DE TAMUJO & Cáceres & & & & & & & & $x$ & & & & & & & & & & & $x$ & Inédito \\
\hline NECROPOLIS DEL MERCADILLO & Cáceres & & & & & & & & $x$ & & 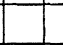 & 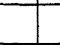 & & & & & & & . & $x$ & Inédito \\
\hline POCITO CHICO & Cádiz & & & & & & \begin{tabular}{|l|}
$x$ \\
\end{tabular} & $x$ & $\mathrm{x}$ & & $x$ & $x$ & & & & & & & $x$ & $x$ & En prensa \\
\hline $\begin{array}{l}\text { GRAZALEMA-SIERRA DEL } \\
\text { ENDRINAL }\end{array}$ & Cádiz & & & & & & & & & & & & & & & & & & & & \begin{tabular}{l|l}
$x$ & En curso
\end{tabular} \\
\hline CUEVA DEL CANUTO & Cádiz & & & & & & & & & & & & & & & & - & $?$ & $?$ & $?$ & En curso \\
\hline EL RETAMAR & Cádiz & & & & & $\mathrm{x}$ & & & & & & & & & & & & $x$ & & 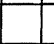 & Estéril \\
\hline \begin{tabular}{|l|} 
FACTORIA P.19 \\
\end{tabular} & Cádiz & & & & & & & & $\mathrm{x}$ & & & & & & & & & & & $\mathrm{x}$ & Estéril \\
\hline $\begin{array}{l}\text { CUEVA DEL LEVANTE-MEDINA } \\
\text { SIDONIA }\end{array}$ & Cádiz & & & & & & & & $x$ & & & & & & & & & & & $\mathrm{x}$ & Estéril \\
\hline DOÑA BLANCA & Cádiz & & & & & & $\mathrm{x}$ & $\mathrm{x}$ & $\mathrm{x}$ & & & & & & & & & & $\mathrm{x}$ & $\mathrm{x}$ & Inédito \\
\hline ABRIGO DE LA PEÑA DEL PERRO & Cantabria & & & $\mathrm{x}$ & $x$ & & & & & & & & & $\mathrm{x}$ & $\mathrm{x}$ & \begin{tabular}{|l|l|l}
$x$ & $x$ \\
\end{tabular} & & & & & López García et al., 1996a \\
\hline CUEVA DEL CASTILLO & Cantabria & & $\mathrm{x}$ & $x$ & & & & 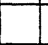 & & & & & \begin{tabular}{|l|l|}
$x$ & $x$ \\
\end{tabular} & $x$ & & & & & 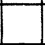 & & Estéril \\
\hline CUEVA DEL SALITRE & Cantabria & & & $\mathrm{x}$ & $\mathrm{x}$ & & & $x$ & & & & & & $\mathrm{x}$ & $x$ & $x$ & $\mathrm{x}$ & & $x$ & & López García, 1981a, 1986b \\
\hline \begin{tabular}{|l} 
NURAGA ARRUBIU \\
\end{tabular} & Cerdeña, Italia & & & & & & & $x$ & & & & & & & & & & & $\mathrm{x}$ & & Inédito \\
\hline NURAGA GASSORU & Cerdeña, Italia & & & & & & & $x$ & & & & & & & & & & & $\mathrm{x}$ & & En curso \\
\hline TUMBA DE GIGANTE STESSEI & Cerdeña, Italia & & & & & & & $\mathrm{x}$ & & & & & & & & & & & $\mathrm{x}$ & & En curso \\
\hline NURAGA DE SU'PUTZU & Cerdeña, Italia & & & & & & & $x$ & & & & & & & & & & & $x$ & & En curso \\
\hline ALARCOS & Ciudad Real & & & & & & & & $\mathrm{x}$ & & & & & & & & & & & $x$ & Estéril \\
\hline \begin{tabular}{|l} 
VALLE DE LOS CABALLEROS \\
\end{tabular} & Ciudad Real & & & & & & & & & & & & & & & & & & & $x$ & \begin{tabular}{l|l}
$x$ & Díaz Fernández, 1994 \\
\end{tabular} \\
\hline \begin{tabular}{|l|} 
RIO FRIO \\
\end{tabular} & Ciudad Real & & & & & & & & & & & & & & & & & & & $\mathrm{x}$ & \begin{tabular}{l|l} 
x & Díaz Fernández, 1994 \\
\end{tabular} \\
\hline NAVALMANZANO & Ciudad Real & & & & & & & & & & & & & & & & & & & $\mathrm{x}$ & \begin{tabular}{l|l}
$\mathrm{x}$ & Díaz Fernández, 1994 \\
\end{tabular} \\
\hline ATEGUA & Córdoba & & & & & & & $x$ & $x$ & & & & & & & & & & $x$ & $x$ & López García, 1986b, 1994; López García y López Sáez,1994a, b \\
\hline
\end{tabular}




\begin{tabular}{|c|c|c|c|c|c|c|c|c|c|c|c|c|c|c|c|c|c|c|c|c|c|}
\hline Yacimiento & \begin{tabular}{|l|} 
Provincia/Departamen. \\
\end{tabular} & PI & PM & PS & M & $\mathrm{N}$ & C & B & PR & \begin{tabular}{l|l}
$\mathbf{R}$ & $\mathbf{E}$ \\
\end{tabular} & EM & \begin{tabular}{l|l}
$\mathrm{EC}$ & $\mathrm{PC}$ \\
\end{tabular} & \begin{tabular}{l|l}
$\mathrm{PG}$ & $\mathrm{OD}$ \\
\end{tabular} & $\mathbf{B} / \mathbf{A}$ & $\mathrm{YD}$ & $\mathrm{PB}$ & BO & $\mathrm{A}$ & SB & SA & \begin{tabular}{|l|l|} 
PR & Referencia \\
\end{tabular} \\
\hline LLANETE DE LOS MOROS & Córdoba & & & & & & & $\mathrm{x}$ & & & & & & & & & & & $\mathrm{x}$ & $\mathrm{x}$ & López García, 1994; López García y López Sáez, 1994a, b, d \\
\hline BARCHIN DEL HOYO & Cuenca & & & & & & & $\mathrm{x}$ & & & & & & & & & & & $\mathrm{x}$ & & Estéril \\
\hline EL RECUENCO & Cuenca & & & & & & & $\mathrm{x}$ & & & & & & & & & $\mathrm{x}$ & & & & López García, 1983a, 1985a, 1986b \\
\hline \begin{tabular}{|l|} 
CASTILLEJO \\
\end{tabular} & Cuenca & & & & & & & $\mathrm{x}$ & & & & & & & & & $x$ & & & & López García, 1983b, 1985a, 1986b \\
\hline VERDELPINO & Cuenca & & & $\mathrm{x}$ & & $\mathrm{x}$ & & & & & & & & $\mathrm{x}$ & $\mathrm{x}$ & ? & & $\mathrm{x}$ & $\mathrm{x}$ & & López Garcia, 1977, 1978, 1985a, 1986b \\
\hline $\begin{array}{l}\text { CERRO DE LA PLAZA DE LOS } \\
\text { MOROS }\end{array}$ & Cuenca & & & & & & & & $x$ & & & & & & & & & & & $x$ & López García, 1985a, 1986b, 1994 \\
\hline VELIKENT & Daguestán, Rusia & & & & & & $\mathrm{x}$ & & & & & & & & & & & & $x$ & & Martín Sánchez et al., 1999 \\
\hline AYMARGE LE REZIL & Gard, Francia & & & & & & & & $\mathrm{x}$ & & $\mathrm{x}$ & $\mathrm{x}$ & & & & & & & . & $x$ & En prensa \\
\hline ARENERO DE LA TORRECILLA & Guadalajara & & & & & & $x$ & $x$ & & & & & & & & & & & $\mathrm{x}$ & & Estéril \\
\hline CASTRO DE LA CORONILLA & Guadalajara & & & & & & & & $\mathrm{x}$ & & & & & & & & & & L & $\mathrm{x}$ & López García, 1982f, 1992c \\
\hline ALARILLA & Guadalajara & & & & & & & $x$ & & & & & & & & & & . & $x$ & & López García, 1986b \\
\hline CUGURON & Haute Garonne, Francia & & & & & & & & & & & & & & & & & $\mathrm{x}$ & $\mathrm{x}$ & $x$ & En prensa \\
\hline LIMOUSIN & Haute Vienne, Francia & & & & & & & & & & & & & & & & & & & & \begin{tabular}{|l|l|} 
x & López Sáez et al., 1998a \\
\end{tabular} \\
\hline GROTTE DE CAMPRAFAUD & Hérault, Francia & & & & & $\mathrm{x}$ & $x$ & & & & & & & & & & & $\mathrm{x}$ & $x$ & & En prensa \\
\hline PAPAUVAS & Huelva & & & & & & $x$ & & & & & & & & & & & & $\mathrm{x}$ & $?$ & López García, 1994; López Garcia y López Sáez, 1994a, b \\
\hline CUEVA DEL MORO & Huesca & & & & & $x$ & & $x$ & & & & & & & & & & & $x$ & $x$ & $\begin{array}{l}\text { López García, 1986b, 1987a, b, 1992, 1994; López García y López } \\
\text { Sáez, 1994c, 1996a }\end{array}$ \\
\hline GABASA & Huesca & & $\mathrm{x}$ & & & & & & & & & $\mathrm{x}$ & $\mathrm{x}$ & & & & & & & L & Azanza et al., 1988 \\
\hline VALLE PEPINO I & Huesca & & & & & & & & $\mathrm{x}$ & & & & & & & & & 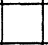 & & $x$ & Estéril \\
\hline CHAVES & Huesca & & & $\mathrm{x}$ & $\mathrm{x}$ & $\mathrm{x}$ & & $\mathrm{x}$ & & & & $\mathrm{x}$ & $\mathrm{x}$ & & & & & $\mathrm{x}$ & & . & López García, 1992a, b; López García y López Sáez, 2000b \\
\hline EL PAJARILLO & Jaén & & & & & & & & $\mathrm{x}$ & $x$ & & & & & & & & & & $x$ & López García y López Sáez, 1996d, 1998 \\
\hline CUEVA DEL NACIMIENTO & Jaén & & & & & $x$ & & & & & & & & & & & & $\mathrm{x}$ & $\mathrm{x}$ & & $\begin{array}{l}\text { López Garcia, 1981c, 1982a, 1986b; López García y López Sáez, } \\
\text { 1994a, b }\end{array}$ \\
\hline CASTELLONES DE CEAL & Jaén & & & & & & & & $x$ & & & & & & & & & & & $x$ & $\begin{array}{l}\text { López García, 1984e, 1985b, 1986b, 1994; López García y López } \\
\text { Sáez, 1994a, b }\end{array}$ \\
\hline MARROQUIES BAJOS & Jaén & & & & & $\mathrm{x}$ & & & & & & & & & & & & $\mathrm{x}$ & & & Inédito \\
\hline PUENTE TABLAS & Jaén & & & & & & $\mathrm{x}$ & & $\mathrm{x}$ & & & & & & & & & & & $x$ & López García, 1994 \\
\hline SEVILLEJA & Jaén & & & & & $\mathrm{x}$ & & $\mathrm{x}$ & & & & & & & & & & $\mathrm{x}$ & $\mathrm{x}$ & & Estéril \\
\hline EL RISQUILLO & Jaén & & & & & & & & & & & & & & & & & & & $x$ & \begin{tabular}{|l|l|}
$x$ & Díaz Fernández, 1994 \\
\end{tabular} \\
\hline PEÑA MIEL & La Rioja & & $x$ & & & & & & & & & $\mathrm{x}$ & $\mathrm{x}$ & & & & & & & & López García, $1987 \mathrm{c}$ \\
\hline LA BAÑA & León & & & & & & & & & & & & & & & & & & & 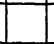 & \begin{tabular}{|l|l|}
$\mathrm{x}$ & Janssen et al., 1996 \\
\end{tabular} \\
\hline ESCABASSES & Lot, Francia & & & & $\mathrm{x}$ & & & $x$ & $\mathrm{x}$ & & $\mathrm{x}$ & & & $\mathrm{x}$ & $\mathrm{x}$ & $\mathrm{x}$ & $\mathrm{x}$ & $\mathrm{x}$ & $\mathrm{x}$ & $\mathrm{x}$ & En prensa \\
\hline CHAO MAZOS & Lugo & & & & & $\mathrm{x}$ & & & & & & & & & & & & & $x$ & & López García y López Sáez, 1993 \\
\hline MEDORRAS DO SAL & Lugo & & & & & $x$ & & & & & & & & & & & & & $\mathrm{x}$ & & López García y López Sáez, 1993 \\
\hline CARABULLA & Lugo & & & & & $\mathrm{x}$ & & & & & & & & & & & & & $\mathrm{x}$ & & López García y López Sáez, 1993 \\
\hline FONTE VILAR & Lugo & & & & & $\mathrm{x}$ & & & & & & & & & & & & & $\mathrm{x}$ & & López García y López Sáez, 1993 \\
\hline CASTILLO DE BARAJAS & Madrid & & - & & & & $\mathrm{x}$ & & $\mathrm{x}$ & & $\mathrm{x}$ & & & & & & & & $\mathrm{x}$ & $x$ & López García, 1997; Macías et al., 1998 \\
\hline PONTÓN DE LA OLIVA & Madrid & & & & & & & & & $\mathrm{x}$ & & & & & & & & & & $\mathrm{x}$ & López García. 1997; Macías et al., 1998 \\
\hline CUEVA DEL SIFÓN & Madrid & & & & & & & & & $?$ & & & & & & & & & & $x$ & López García. 1997; Macías et al., 1998 \\
\hline CASERIO DE PERALES & Madrid & & & & & & & $\mathrm{x}$ & & & & & & & & & & & & $x$ & López García, 1997; Macias et al., 1998 \\
\hline
\end{tabular}




\begin{tabular}{|c|c|c|c|c|c|c|c|c|c|c|c|c|c|c|c|c|c|c|c|c|c|}
\hline Yacimiento & Provincia/Departamen. & $\mathrm{PI}$ & PM & PS & $\mathrm{M}$ & $\mathrm{N}$ & $\mathrm{C}$ & $\mathrm{B}$ & PR & \begin{tabular}{|l|ll}
$\mathbf{R}$ \\
\end{tabular} & EM $1 \mathrm{~F}$ & \begin{tabular}{|l|l|l}
$E C$ & $\mathbf{P}$ \\
\end{tabular} & \begin{tabular}{l|l|l} 
PG & OD \\
\end{tabular} & $\mathrm{B} / \mathrm{A}$ & YD & PB & BO & 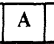 & SB & \begin{tabular}{|l|l}
$\mathrm{SA}$ & $\mathrm{I}$ \\
\end{tabular} & \begin{tabular}{l|l} 
PR & Referencia \\
\end{tabular} \\
\hline EL VENTORRO & Madrid & & & & & & & $x$ & & & & & & & & & & & $\mathrm{x}$ & & $\begin{array}{l}\begin{array}{l}\text { López García, 1985a, 1986b, 1997; López García y Arnanz, } 1994 \\
\text { Macias et al., 1998 }\end{array} \\
\end{array}$ \\
\hline ECCE HOMO & Madrid & & & & & & $\mathrm{x}$ & $\mathrm{x}$ & $x$ & & & & & & & & & & $\mathrm{x}$ & $x$ & López García, 1997; Macías et al., 1998 \\
\hline LAS FRONTERAS & Madrid & & & & & & & & $\mathrm{x}$ & & & & & & & & & & & $x$ & López García, 1997; Macías et al., 1998 \\
\hline VILLAVERDE-91 & Madrid & & & & & & & & $x$ & & & & & & & & & & & $x$ & López García, 1997; Macías et al., 1998 \\
\hline FABRICA DE LADRILLOS & Madrid & & & & & & & & $x$ & & & & & & & & & & & $x$ & López García, 1997; Macías et al., 1998 \\
\hline VALDELAZARO & Madrid & & & & & & & $\mathrm{x}$ & & & & & & & & & & & $\mathrm{x}$ & $\mathrm{x}$ & López García, 1997 \\
\hline SOLAR 3M & Madrid & & & & & & & & $\mathrm{x}$ & & & & & & & & & & & $\mathrm{x}$ & López García, 1997 \\
\hline OCISA-ESGARAVITA & Madrid & & & & & & & & $\mathrm{x}$ & & & & & & & & & & & $\mathrm{x}$ & López García, 1997 \\
\hline FUENTE DEL JUNCAL & Madrid & & & & & & & & $\mathrm{x}$ & & & & & & & & & & & $x$ & López García, 1997 \\
\hline VERONA II & Madrid & & & & & & & $x$ & $\mathrm{x}$ & & & & & & & & & & $?$ & $\mathrm{x}$ & López García, 1997 \\
\hline CANTERA DE LA FLAMENCA & Madrid & & & & & & & $\mathrm{x}$ & & & & & & & & & & & & $\mathrm{x}$ & López García, 1997 \\
\hline LAGUNA DE LIRIALON & Madrid & & & & & & & & & & & & & & & & & & & $x$ & López García, 1997 \\
\hline FONDOS DE CABAÑA & Madrid & & & & & & & $x$ & & & & & & & & & $\mathrm{x}$ & & & 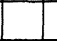 & López García, 1983c, 1985a, 1986b \\
\hline LAGUNA DE MARIA PASCUALA & Madrid & & & & & & & & & & & & & & & & & & & $\mathrm{x}$ & López García, 1997; Macias et al., 1998 \\
\hline LAGUNA DE AJALVIR & Madrid & & & & & & & & & & & & & & & & & & - & $x$ & López García, 1997; Macias et al., 1998 \\
\hline PEÑALARA & Madrid & & & & & & & & & & & & & & & & & & $\mathrm{x}$ & $\mathrm{x}$ & Jiménez et al., 1985; López García, 1997 \\
\hline VALDESQUI & Madrid & & & & & & & & & & & & & & & & & & $?$ & $x$ & Jiménez et al., 1985; López García, 1997 \\
\hline LA BUITRERA & Madrid & & & & & & & & & & & & & & & & & & $?$ & $\mathrm{x}$ & Jiménez et al., 1985; López García, 1997 \\
\hline VALDETORRES DEL JARAMA & Madrid & & & & & & & & & $x$ & & & & & & & & & & $\mathrm{x}$ & López García, 1985a, 1986b, 1994 \\
\hline LA PINILLA & Madrid & & & & & & & & & & & ? & $?$ & & & & & & & & Estéril \\
\hline ARENERO DEL SOTO & Madrid & & & & & & $\mathrm{x}$ & $\mathrm{x}$ & & & & & & & & & & & $\mathrm{x}$ & 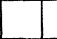 & Estéril \\
\hline LAS MATILLAS & Madrid & & & & & & & & $x$ & & . & & & & & & & & 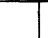 & $\mathrm{x}$ & Estéril \\
\hline GOZQUEZ & Madrid & & & & & & $\mathrm{x}$ & & & & $\mathrm{x}$ & & & & & & & & $\mathrm{x}$ & $\mathrm{x}$ & En prensa \\
\hline CUEVA DE LA PALOMA & Málaga & & & & & $x$ & & & & & & & & & & & & $\mathrm{x}$ & & & Estéril \\
\hline HOYO DE LA MINA & Málaga & & & & & $\mathrm{x}$ & & & & & & & & & & & & $\mathrm{x}$ & & & En curso \\
\hline BAJONCILLO & Málaga & & $\mathrm{x}$ & $\mathrm{x}$ & $\mathrm{x}$ & $\mathrm{x}$ & & & & & & $\mathrm{x}$ & \begin{tabular}{c|c}
$x$ & $x$ \\
\end{tabular} & $\mathrm{x}$ & $\mathrm{x}$ & $\mathrm{x}$ & $\mathrm{x}$ & $\mathrm{x}$ & & & En curso \\
\hline BINIAU NOU & Menorca & & & & & & & & $\mathrm{x}$ & & & & & & & & & & $x$ & & En prensa \\
\hline CUEVA DEL CALOR & Murcia & & & & & \begin{tabular}{|l|l}
$\mathrm{x}$ \\
\end{tabular} & $\mathrm{x}$ & $\mathrm{x}$ & $\mathrm{x}$ & & & & & & & & & $\mathrm{x}$ & $x$ & $\mathrm{x}$ & López García, 1988a, 1991 \\
\hline CORTIJO DE ANDREVIIAA & Murcia & & & & & & $\mathrm{x}$ & & & & & & & & & & & 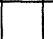 & $\mathrm{x}$ & & López García, 1991 \\
\hline EL MILANO & Murcia & & & & & & $\mathrm{x}$ & & & & & & & & & & & $x$ & & & López García, 1988a, 1991 \\
\hline LA PRESA DEL ESPARRAGAL & Murcia & & & & & & & & $\mathrm{x}$ & & & & & & & & & & & $\mathrm{x}$ & López García, 1991 \\
\hline LOS MOLINICOS & Murcia & & & & & & & $\mathrm{x}$ & $x$ & & & & & & & & & & $x$ & $x$ & López Garcia, 1991 \\
\hline EL PRADO & Murcia & & & & & & $\mathrm{x}$ & & & $x$ & & & & & & & & & $\mathrm{x}$ & & López García, 1988a, 1991 \\
\hline ARROYO TERCERO & Murcia & & & & & & & & $\mathrm{x}$ & & & & & & & & & & & $x$ & López Garcia, 1991 \\
\hline $\begin{array}{l}\text { EL ABRIGO DE LOS } \\
\text { CARBONEROS } \\
\end{array}$ & Murcia & & & & & & $\mathrm{x}$ & & & & & & & & & & & & $\mathrm{x}$ & & López García, 1988a, 1991 \\
\hline ABAUNTZ & Navarra & & & $x$ & $x$ & \begin{tabular}{|l|l}
$x$ & -1 \\
\end{tabular} & $\mathrm{x}$ & & & $x$ & & & $x$ & & & $\mathrm{x}$ & $\mathrm{x}$ & $x$ & $x$ & $x$ & López García, 1982b, 1986b, 1987a; Utrilla et al. 1986 \\
\hline LA PEÑA & Navarra & & & & $\mathrm{x}$ & \begin{tabular}{l|l}
$x$ \\
\end{tabular} & $\mathrm{x}$ & $\mathrm{x}$ & & & & & & & & $\mathrm{x}$ & $\mathrm{x}$ & $x$ & $x$ & & López García, 1987a \\
\hline CASTILLAR DE MENDAVIA & Navarra & & & & & & & & $\mathrm{x}$ & & & & & & & & & & & $\mathrm{x}$ & López García, 1993 \\
\hline
\end{tabular}




\begin{tabular}{|c|c|c|c|c|c|c|c|c|c|c|c|c|c|c|c|c|c|c|}
\hline Yacimiento & Provincia/Departamen. & PI & \begin{tabular}{|l|l|l|l|l|l|l} 
PM & P \\
\end{tabular} & \begin{tabular}{l|l} 
PS & M \\
\end{tabular} & $1 \mathrm{~N}$ & \begin{tabular}{|c|}
$\mathrm{C}$ \\
\end{tabular} & B & \begin{tabular}{|l|l}
$\mathbf{P R}$ & $\mathbf{R}$ \\
\end{tabular} & \begin{tabular}{|l|l|}
$\mathbf{R}$ & EM \\
\end{tabular} & 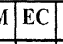 & \begin{tabular}{|l|l|l|} 
PG & OD \\
\end{tabular} & B/A & \begin{tabular}{|l|l|l}
$\mathrm{YD}$ & $\mathrm{P}$ \\
\end{tabular} & 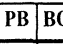 & \begin{tabular}{l|l}
30 & $\mathrm{~A}$ \\
\end{tabular} & SB & SA & \begin{tabular}{|l|l|} 
PR & Referencia \\
\end{tabular} \\
\hline GORNI-1 & Orenburg, Rusia & & & & & & $\mathrm{x}$ & & & $\mathrm{x}$ & & & & & & $\mathrm{x}$ & $\mathrm{x}$ & \begin{tabular}{|l|l|} 
x & López García et al., 1996b; Vicent et al., 2000 \\
\end{tabular} \\
\hline GORNI-2 & Orenburg, Rusia & & & & & & $\mathrm{x}$ & & & $\mathrm{x}$ & & & & & & $\mathrm{x}$ & $\mathrm{x}$ & \begin{tabular}{l|l}
$\mathrm{x}$ & Vicent $e t$ al., 2000 \\
\end{tabular} \\
\hline NOVIENKI Y ACIMIENTO & Orenburg, Rusia & & & & & & $\mathrm{x}$ & & & $x$ & & & & & & $\mathrm{x}$ & $x$ & \begin{tabular}{c|l|}
$\mathrm{x}$ & Vicent et al., 2000 \\
\end{tabular} \\
\hline NOVIENKI TERRAZA & Orenburg, Rusia & & & & & & & & & & & & & & & & $\mathrm{x}$ & \begin{tabular}{|l|l|}
$\mathrm{x}$ & Vicent et al., 2000 \\
\end{tabular} \\
\hline NOVIENKI TURBERA & Orenburg, Rusia & & & & & & & & & & & & & & & $\mathrm{x}$ & $x$ & \begin{tabular}{l|l|}
$\mathrm{x}$ & Vicent et al., 2000 \\
\end{tabular} \\
\hline CASA RUSA & Orenburg, Rusia & & & & & & & & & $\mathrm{x}$ & & & & & & $\mathrm{x}$ & $\mathrm{x}$ & \begin{tabular}{l|l|}
$\mathrm{X}$ & Vicent et al., 2000 \\
\end{tabular} \\
\hline ESCOMBRERAS DE GORNI & Orenburg, Rusia & & & & & & & & & $\mathrm{x}$ & & & & & & & $\mathrm{x}$ & \begin{tabular}{l|l|}
$\mathrm{x}$ & Vicent et al., 2000 \\
\end{tabular} \\
\hline O FIXÓN & Pontevedra & & & & & & $\mathrm{x}$ & & & & & & & & & $\mathrm{x}$ & & López García, 1984d, 1986b \\
\hline LAVAPES & Pontevedra & & & & & $\mathrm{x}$ & & & & & & & & & & $\mathrm{x}$ & $x$ & López García, 1982d, 1984c, 1986b \\
\hline LESCAR LALA NUSSE & $\begin{array}{l}\text { Pyrénées Atlantiques, } \\
\text { Francia } \\
\end{array}$ & & & & & & & & $\mathrm{x}$ & & & & & & & & $x$ & Inédito \\
\hline OLORON & $\begin{array}{c}\text { Pyrénées Atlantiques, } \\
\text { Francia } \\
\end{array}$ & & & & & & & & \begin{tabular}{l|l|}
$x$ & $x$ \\
\end{tabular} & & & & & & & & $\mathrm{x}$ & López Sáez et al, 1998a \\
\hline ARTXILONDO & $\begin{array}{l}\text { Pyrénées Atlantiques, } \\
\text { Francia } \\
\end{array}$ & & & & & & & & & & & & & \begin{tabular}{l|l}
$x$ & $x$
\end{tabular} & $\mathrm{x}$ & $\mathrm{x}$ & $x$ & En prensa \\
\hline DOLMEN CREU DE LA FALIBE & $\begin{array}{c}\text { Pyrénées Orientales, } \\
\text { Francia } \\
\end{array}$ & & & & $\mathrm{x}$ & & $?$ & & & & & & & & $\mathrm{x}$ & $x$ & & López Sáez et al., 1998a \\
\hline LA SERRAT D'EN JACQUES & $\begin{array}{l}\text { Pyrénées Orientales, } \\
\text { Francia } \\
\end{array}$ & & & & $\mathrm{x}$ & & & & & & & & & & $\mathrm{x}$ & & & Inédito \\
\hline DOLMEN COL LLOSA & $\begin{array}{c}\text { Pyrénées Orientales, } \\
\text { Francia } \\
\end{array}$ & & & & $\mathrm{x}$ & & & & & & & & & & $\mathrm{x}$ & & & Inédito \\
\hline LES RIERES & $\begin{array}{l}\text { Pyrénées Orientales, } \\
\text { Francia } \\
\end{array}$ & & & & $\mathrm{x}$ & & & & & & & & & & $\mathrm{x}$ & & & Inédito \\
\hline L'OLIVIA D'EN DAVID & $\begin{array}{l}\text { Pyrénées Orientales, } \\
\text { Francia }\end{array}$ & & & & $\mathrm{x}$ & & & & & & & & & & $\mathrm{x}$ & & & Inédito \\
\hline MAURA & $\begin{array}{l}\text { Pyrénées Orientales, } \\
\text { Francia } \\
\end{array}$ & & & & & & & & & & & & & & & $\mathrm{x}$ & $x$ & Inédito \\
\hline PLA DE L'ORRI & $\begin{array}{l}\text { Pyrénées Orientales, } \\
\text { Francia } \\
\end{array}$ & & & & & & & & & & & & & & $\mathrm{x}$ & $\mathrm{x}$ & $x$ & Inédito \\
\hline EYNE & $\begin{array}{c}\text { Pyrénées Orientales, } \\
\text { Francia } \\
\end{array}$ & & & & & & & & & & & & & & & $\mathrm{x}$ & $\mathrm{x}$ & Inédito \\
\hline LAC DU LAUZON & Rhône, Francia & & & & & & & & & & & & & & $\mathrm{x}$ & $\mathrm{x}$ & $\mathrm{x}$ & Inédito \\
\hline LES CAVENES DE CABACO & Salamanca & & & & & & & $x$ & $\mathrm{x}$ & & & & & & & & $x$ & Inédito \\
\hline FUENTE DE LA MORA FMC & Salamanca & & & & & & & $\mathrm{x}$ & $\mathrm{x}$ & & & & & & & & $\mathrm{x}$ & Inédito \\
\hline LA SOLANA & Salamanca & & & & & & & $\mathrm{x}$ & & & & & & & & & $\mathrm{x}$ & Inédito \\
\hline EL CHORRITO & Salamanca & & & & & & & $\mathrm{x}$ & & & & & & & & & $\mathrm{x}$ & Inédito \\
\hline LA CORVERA & Salamanca & & & & & & & $\mathrm{x}$ & & & & & & & & & $\mathrm{x}$ & Inédito \\
\hline LA VAQUERA & Segovia & & & & $\mathrm{x}$ & & $\mathrm{x}$ & $\mathrm{x}$ & & & & & & & . & . & & López García et al., 1997a \\
\hline \begin{tabular}{|l|l|} 
BURGOMILLODO \\
\end{tabular} & Segovia & & & & & & & & & & & $\mathrm{x}$ & \begin{tabular}{l|l}
$x$ & $x$ \\
\end{tabular} & \begin{tabular}{l|l}
$x$ & $x$ \\
\end{tabular} & $\mathrm{x}$ & $\mathrm{x}$ & $\mathrm{x}$ & En prensa \\
\hline PUENTE DE VILASECA & Segovia & & & & & & & & & & & & & & $x$ & & & En curso \\
\hline LOS MOLARES & Sevilla & & & & & $\mathrm{x}$ & & & & & & & & & & $\mathrm{x}$ & $\mathrm{x}$ & López García, 1994; López García y López Sáez, 1994a, 1997 \\
\hline CUEVA DE LOS COVACHOS & Sevilla & & & & & $\mathrm{x}$ & $\mathrm{x}$ & & & & & & & & & $\mathrm{x}$ & & En prensa \\
\hline $\begin{array}{l}\text { TRAVERTINOS ALMADEN DE LA } \\
\text { PLATA }\end{array}$ & Sevilla & & & & & & & & & & & & & & & $x$ & & En prensa \\
\hline
\end{tabular}




\begin{tabular}{|c|c|c|c|c|c|c|c|c|c|c|c|c|c|c|c|c|c|c|c|}
\hline Yacimiento & Provincia/Departamen. & \begin{tabular}{|l|l|l|l|} 
PI & 1 \\
\end{tabular} & \begin{tabular}{l|l}
$\mathrm{PM}$ & $\mathrm{PS}$ \\
\end{tabular} & \begin{tabular}{l|l} 
PS & $M$
\end{tabular} & $\mathrm{~N}$ & C & \begin{tabular}{l|l} 
B & 1 \\
\end{tabular} & \begin{tabular}{|l|l|} 
PR & 1 \\
\end{tabular} & \begin{tabular}{l|l}
$\mathbf{R}$ & $\mathbf{I}$ \\
\end{tabular} & \begin{tabular}{|l|l|l|l|l}
$E M$ & $E$ \\
\end{tabular} & \begin{tabular}{l|l}
$\mathrm{EC}$ & $\mathrm{P}$ \\
\end{tabular} & \begin{tabular}{l|l|l}
$\mathrm{PG}$ & $\mathrm{OD}$ \\
\end{tabular} & \begin{tabular}{|l|l|}
$D$ & $B / A$ \\
\end{tabular} & YD & \begin{tabular}{|l|l|} 
PB & $B O$ \\
\end{tabular} & \begin{tabular}{|l|l|}
0 & A \\
\end{tabular} & SB & \begin{tabular}{|l|l|} 
SA & P \\
\end{tabular} & \begin{tabular}{l|l|} 
PR & Referencia \\
\end{tabular} \\
\hline $\begin{array}{l}\text { CANTERA ROMANA DE } \\
\text { ALMADEN }\end{array}$ & Sevilla & & & & & & & & $\mathrm{x}$ & & & & & & & & & \begin{tabular}{l|l}
$x$ & \\
\end{tabular} & En prensa \\
\hline BABIDOL & Somonimo, Polonia & & & & & & & $\mathrm{x}$ & & & & & & & & & & $x$ & Inédito \\
\hline PARPANTIQUE & Soria & & & & & & $\mathrm{x}$ & & & $\mathrm{x}$ & & & & & & & $\mathrm{x}$ & $\mathrm{x}$ & López García y López Sáez, 1999 \\
\hline TOLMOS DE CARACENA & Soria & & & & & & $\mathrm{x}$ & & & & & & & & $x$ & 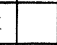 & & & López García, 1981d, e, 1985a, 1986b \\
\hline CASTILLEJO DE NUMANCIA & Soria & & & & & $\mathrm{x}$ & & $\mathrm{x}$ & $\mathrm{x}$ & & & & & & & & $\mathrm{x}$ & $\mathrm{x}$ & Inédito \\
\hline CASTILMONTAN & Soria & & & & & & & $\mathrm{x}$ & & & & & & & & & & $\mathrm{x}$ & Estéril \\
\hline FUENTE BLANQUILLA & Soria & & & & $\mathrm{x}$ & & & & & & & & & & & $x$ & & & Estéril \\
\hline PENINJ & Tanzania & $x$ & & & & & & & & & & $x$ & & & & & & & En prensa \\
\hline BOTIQUERIA DELS MOROS & Teruel & & & $\mathrm{x}$ & $\mathrm{x}$ & & & & & & & & & & & $x$ & $?$ & & $\begin{array}{l}\text { López García 1992a, b; López García et al. 1991; López García y } \\
\text { López Sáez, 2000b }\end{array}$ \\
\hline ELS SECANS & Teruel & & & $\mathrm{x}$ & $\mathrm{x}$ & & & & & & & & & & & $\mathrm{x}$ & $\mathrm{x}$ & & López García, 1992a, b; López García y López Sáez, 1996b, 2000b \\
\hline MONTÓN DE TIERRA-GRIEGOS & Teruel & & & & & & $x$ & & & & & & & & & & $\mathrm{x}$ & $\mathrm{x}$ & López García y López Sáez, 1996c \\
\hline EL PONTET & Teruel & & & $\mathrm{x}$ & $\mathrm{x}$ & & & & & & & & & & & $x$ & & & López García, 1992a,b;López García y López Sáez, 2000b \\
\hline ALONSO NORTE & Teruel & & & & $\mathrm{x}$ & & & & & & & & & & & $\mathrm{x}$ & & & López García, 1992a,b \\
\hline CUEVA DEL COSCOJAR & Teruel & & & & $\mathrm{x}$ & & $\mathrm{x}$ & $x$ & & & & & & & & $\mathrm{x}$ & $\mathrm{x}$ & $\mathrm{x}$ & López García, 1986b, 1987a,1992a, b, 1994 \\
\hline HOYA QUEMADA & Teruel & & & & & & $x$ & & & & & & & & & & $\mathrm{x}$ & & Picazo et al. 1997 \\
\hline DOLMEN DE AZUTÁN & Toledo & & & & $\mathrm{x}$ & & & & & & & & & & & $\mathrm{x}$ & & & López García y López Sáez, 2000a \\
\hline HUECAS-EL CASTILLEJO & Toledo & & & & & & & $\mathrm{x}$ & & & & & & & & & & $\mathrm{x}$ & Inédito \\
\hline MELQUE & Toledo & & & & & & & & & $x$ & & & & & & 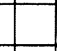 & & $\mathrm{x}$ & Macías Rosado y López García, 1999 \\
\hline LA SARSA & Valencia & & & & $\mathrm{x}$ & & & & & & & & & & & $x$ & & & $\begin{array}{l}\text { López García, 1986b; López García y Molero, 1984; Asquerino et al., } \\
1998\end{array}$ \\
\hline ROQUEBRUNE SUR ARGENS & Var, Francia & & & & & & & $\mathrm{x}$ & \begin{tabular}{l|l}
$\mathrm{x}$ & $\mathrm{B}$ \\
\end{tabular} & \begin{tabular}{l|l}
$\mathrm{x}$ & $\mathrm{z}$ \\
$\mathrm{y}$
\end{tabular} & $\mathrm{x}$ & & & & & & & \begin{tabular}{l|l}
$x$ & $x$ \\
\end{tabular} & \begin{tabular}{|l|l|} 
x & López Sáez et al.,1998a \\
\end{tabular} \\
\hline LA COMBETTE & Vaucluse, Francia & & $\mathrm{x}$ & & & & & & & 4 & & $\mathrm{x}$ & & & & 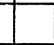 & & $\rightarrow$ & López Sáez et al., 1998a, b \\
\hline LES PETITES BATIES & Vaucluse, Francia & & & & $\mathrm{x}$ & & & & & & & & & & & $\mathrm{x}$ & & & López Sáez et al., 1998a \\
\hline LA PALUD-LES DEVES & Vaucluse, Francia & & & & & & & $\mathrm{x}$ & $\mathrm{x}$ & $\mathrm{x}$ & & & & & & & & $\mathrm{x}$ & López Sáez et al., 1998a \\
\hline BOLLENE LES BARTRAS & Vaucluse, Francia & & & & & & & $\mathrm{x}$ & & $\mathrm{x}$ & $\mathrm{x}$ & & & & & & & $\mathrm{x}$ & En prensa \\
\hline MONDRAGON LES BRASSIERES & Vaucluse, Francia & & & & & & & $\mathrm{x}$ & & $\mathrm{x}$ & $\mathrm{x}$ & & & & & & & $\mathrm{x}$ & En prensa \\
\hline PIERRELATE LES MALALONES & Vaucluse, Francia & & & & & & & $x$ & & $\mathrm{x}$ & $\mathrm{x}$ & & & & & & & $\mathrm{x}$ & En prensa \\
\hline MONDRAGON LE DUC & Vaucluse, Francia & & & & & & & $x$ & & $\mathrm{x}$ & $\mathrm{x}$ & & & & & & & $\mathrm{x}$ & En prensa \\
\hline LA MOTTE DU RHONE & Vaucluse, Francia & & & & & & & $\mathrm{x}$ & & $\mathrm{x}$ & $\mathrm{x}$ & & & & & & & $x$ & En prensa \\
\hline AMBRUSSUM 1 & Vaucluse, Francia & & & & & & & $\mathrm{x}$ & & $\mathrm{x}$ & $x$ & & & & & & & $x$ & En prensa \\
\hline AMBRUSSUM 2 & Vaucluse, Francia & & & & & & & $x$ & & $\mathrm{x}$ & $\mathrm{x}$ & & & & & & & $x$ & En prensa \\
\hline LA PALUD LES GIRARDES 2 & Vaucluse, Francia & & & & & & & $x$ & & $\mathrm{x}$ & $\mathrm{x}$ & & & & & & & $\mathrm{x}$ & En prensa \\
\hline SAINT ROMAIN DE JALIONAS t. 2 & Vaucluse, Francia & & & & & & & $x$ & & $\mathrm{x}$ & $\mathrm{x}$ & & & & & & & $x$ & En prensa \\
\hline LAGUNA DE CERIPAS & Zamora & & & & & & & & & & & & & & & & & $\mathrm{x}$ & Gómez Ferreras et al., 1996 \\
\hline LAGUNA DEL VILLARDON & Zamora & & & & & & & & & & & & & & & & & $\mathrm{x}$ & Inédito \\
\hline MAJALADARES & Zaragoza & & & & & & $\mathrm{x}$ & & & & & & & & & & $\mathrm{x}$ & & López García, 1992a,b \\
\hline LA LOMA DE LOS BRUNOS & Zaragoza & & & & & & & $\mathrm{x}$ & & & & & & & & & & & López García, 1982c, 1986b, 1987a, 1994 \\
\hline
\end{tabular}




\section{BIBLIOGRAFÍA}

Aira, M.J.; SaA, P. y López García, P. (1992): “Cambios del paisaje durante el Holoceno: análisis de polen en turberas (Galicia, España)". Revue de Paléobiologie 11: 243-254.

Asquerino, M.D.; López García, P.; Molero, G.; Sevilla, P.; Aparicio, M.T. y Ramos, M.A. (1998): "Cova de la Sarsa (Bocairent, Valencia), Sector II: Gatera". Recerques del Museu d'Alcoi 7: 47-88.

Azanza, B.; Baldellou, V.; Chuchi, J.A.; LóPez García, P.; Montes, L. y Utrilla, P. (1988): "Cronoestratigrafía de la cueva musteriense de Los Moros (Gabasa, Huesca)". Cuaternario y Geomorfología 2: 1-25.

Cacho, C.; Fumanal, P.; López García, P. y López, N. (1983): "Contribution du Tossal de la Roca à la chronostratigraphie du Paléolithique supérieur final dans la région de Valence". Rivista di scienze preistoriche 38 (1-2): 69-90.

Cacho, C.; Fumanal, M.P.; López García, P.; López Sáez, J.A.; Pérez Ripoll, M.; Martínez Valle, R.; Uzquiano, P.; Arnanz, A.; Sánchez Marco, A.; Sevilla, P.; Morales, A.; Roselló, E.; Garralda, M.D. y García Carrillo, M. (1995): "El Tossal de la Roca (Vall d'Alcalá, Alicante). Reconstrucción paleoambiental y cultural de la transición del Tardiglaciar al Holoceno inicial". Recerques del Museu d'Alcoi 4: 11-101.

Cacho, C.; Fumanal, P.; López García, P.; López Sáez, J.A.; Arnanz, A.; Uzquiano, P.; Pérez Ripoll, M.; Martínez, R.; Sánchez Marco, A.; Morales, A. y Roselló, E. (1998a): "The transition from Magdalenian to Epipaleolithic in the Spanish Mediterranean: El Tossal de la Roca". Préhistoire Européenne 12: $155-$ 170.

Cacho, C.; Fumanal, M.P.; López García, P.; López Sáez, J.A.; Arnanz, A.; Uzquiano, P.; Pérez Ripoll, M.; SánChez Marco, A.; Morales, A. y Roselló, E. (1998b): "The transition from Magdalenian to Epipaleolithic in the Spanish Mediterranean: El Tossal de la Roca". En C. Giunchi (ed.): Proceedings of the XII Congress of the International Union of Prehistoric and Protohistoric Sciences 2. A.B.A.C.O. Forli, Italia: 635-640.

DíAZ FERNÁNDEZ, P. (1994): "Relations between modern pollen rain and mediterranean vegetation in Sierra Madrona (Spain)". Review of Palaeobotany and Palynology 82: 113-125.

Gómez Ferreras, C.; López García, P. y López Sáez, J.A. (1996): "Dinámica de la vegetación de las lagunas de Villafáfila (Zamora) durante el Holoceno reciente". En B. Ruiz Zapata (ed.): Estudios Palinológicos. Universidad de Alcalá de Henares. Alcalá de Henares (Madrid): 57-61.

Gutiérrez Bustillo, M. (1994): "Bibliografía palinológica española. Años 1988-1992 y adiciones". Lazaroa 14: 139-165.

JANSSEN, C.R.; López SÁEz, J.A. y López García, P. (1996):
"Representatividad de la vegetación de la Sierra de Cabrera Baja (León) en base al estudio de la lluvia polínica actual". En B. Zapata (eds.): Estudios Palinológicos. Universidad de Alcalá de Henares, Alcalá de Henares (Madrid): 167-170.

JiMÉNEZ, R.; LóPEZ MARTíNEZ, J.; LóPEZ GARCÍA, P. y IbÁÑEZ, J.J. (1985): "Contribución al conocimiento de las formaciones superficiales turbosas en las Sierras de Guadarrama y Ayllón. Análisis polínicos". Actas $1^{a}$ Reunión del Cuaternario Ibérico (G.E.T.C.) II: 213-224. Lisboa.

LóPEz GarCíA, P. (1977): “Apéndice II. Análisis polínico de Verdelpino (Cuenca)". Trabajos de Prehistoria 34: 8283.

- (1978): "Resultados polínicos del Holoceno en la Península Ibérica". Trabajos de Prehistoria 35: 9-44.

- (1981a): "Los pólenes de la Cueva de El Salitre". Trabajos de Prehistoria 38: 93-96.

- (1981b): "Análisis polínico del yacimiento de Los Azules (Cangas de Onís, Oviedo)". Botánica Macaronésica 8-9: 243-248.

- (1981c): "Análisis polínico de la Cueva del Nacimiento". Trabajos de Prehistoria 38: 146-148.

- (1981d): "La palinología: una ciencia auxiliar de la Arqueología". Arevacon 3: 8-10.

- (1981e): "Estudios polínicos del yacimiento de los Tolmos de Caracena (Soria)". En A. Jimeno (ed.): Los Tolmos de Caracena. Excavaciones Arqueológicas en España 134. Madrid: 335-338.

- (1982a): "Diagrama polínico del yacimiento de la Cueva del Nacimiento (Pontones, Jaén)". En Le Néolithique Ancien Mediterranéen, Actes du Colloque International de Préhistoire (Montpellier 1981). Archéologie en Languedoc, $\mathrm{n}^{\circ}$ special: $250-251$.

- (1982b): "Abauntz: Análisis polínico". Trabajos de Arqueología Navarra 3: 355-358.

- (1982c): "Estudio paleobotánico de la Loma de los Brunos". En J.J. Eiroa (ed.): La Loma de los Brunos y los Campos de Urnas del Bajo Aragón. Institución Fernando el Católico, Zaragoza: 100-108.

- (1982d): "Análisis polínico del yacimiento de Lavapes (Cangas de Morrazo, Pontevedra)". Museo de Pontevedra: 3-7.

- (1982e): "Polen para arqueólogos". Revista de Arqueología 21: 8-13.

- (1982f): "Estudio de los restos vegetales del Castro de la Coronilla". Noticiario Arqueológico Hispánico 14: 89-90.

- (1983a): "Estudio polínico del yacimiento de 'El Recuenco' (Cervera del Llano), Cuenca". Homenaje al Profesor M. Almagro Basch. Ministerio de Cultura. Madrid 2: 45-48.

- (1983b): "Análisis polínico del Cerro de Castillejo (La Parra de las Vegas)". Noticiario Arqueológico Hispanico 16: 215-217.

- (1983c): "Análisis polínico de cinco "fondos de cabaña" del km 7 dcha. de la carretera de San Martín de la 
Vega (T.M. Getafe, Madrid)". Estudios de Prehistoria y Arqueología Madrileñas 2: 267-269.

- (1984a): "Estudio polínico del yacimiento de El Raso de Candeleda (Avila)". En F. Fernández Gómez: El Raso de Candeleda. Aportaciones al conocimiento de la Edad del Hierro en la zona media del Sistema Central, II. Diputación Provincial de Avila, Institución Gran Duque de Alba. Avila: 918.

- (1984b): “Aplicaciones de la Palinología a la Prehistoria: métodos utilizados y resultados". Primeras Jornadas de Metodología de Investigación Prehistórica. Soria 1981. Ministerio de Cultura. Madrid: 309-317.

- (1984c): "Estudio polínico de los sedimentos del yacimiento de Lavapés". Pontevedra Arqueológica 1: 179188.

- (1984d): “Análisis polínicos de los sedimentos arqueológicos del yacimiento de O Fixón (Viño, Hio, Campos de Morazo)". Pontevedra Arqueológica 1: 145-147.

- (1984e): “Análisis polínico”. En T. Chapa, M. Fernández, J. Pereira y A. Ruiz: Análisis económico y territorial de Los Castellones de Ceal (Jaén). Arqueología Espacial. Coloquio sobre Distribución y Relaciones entre los Asentamientos 4: 223-240.

- (1985a): "Resultados de análisis polínicos del Holoceno en la Meseta española procedentes de yacimientos arqueológicos". Anales de la Asociación de Palinólogos de Lengua Española 2: 283-288.

- (1986a): "La palinología arqueológica". Revista Interdisciplinar de Métodos y Metodología Arqueológica I (1): 23-27.

- (1986b): "Estudio palinológico del Holoceno español a través del análisis de yacimientos arqueológicos". Trabajos de Prehistoria 43: 143-158.

- (1987a): "Datos polínicos del Holoceno de Navarra y Aragón”. En J. Civis y M. Valle (eds.): Actas del VI Simposio de la Asociación de Palinólogos de Lengua Española A.P.L.E: 315-320. Salamanca.

- (1987b): "Estudio polínico del yacimiento de la Cueva del Moro (Olvena, Huesca)". Cuaternario y Geomorfología 2: 1-12.

- (1987c): "Estudio polínico del yacimiento de Peña Miel. En P. Utrilla, (ed.): El yacimiento de Peña Miel. Excavaciones Arqueológicas en España. Madrid: 149-151.

- (1988a): "Estudio polínico de seis yacimientos del Sureste español". Trabajos de Prehistoria 45: 335-345.

- (1988b): "Análisis palinológico de los sedimentos de Cueva Ambrosio (Almería)". En S. Ripoll: La cueva de Ambrosio (Vélez-Blanco, Almería) y su posición cronoestratigráfica en el Mediterráneo occidental. British Archaeological Reports 462. Oxford: 129-134.

- (1989): "La palinología como Ciencia aplicada a los sedimentos arqueológicos". Trabajos de Prehistoria 49: 395-404.

- (1991): "Estudios Palinológicos". En P. López García (ed.): El cambio cultural del IV al II milenios a.C. en la comarca Noroeste de Murcia I. CSIC. Madrid:213-237.
- (1992a): "Análisis polínicos de cuatro yacimientos arqueológicos situados en el Bajo Aragón”. En P. Utrilla, (coord.): Aragón/Litoral mediterráneo: intercambios culturales durante la Prehistoria. Institución Fernando el Católico. Zaragoza: 235-242.

- (1992b): "Pollen analyses from archaeological sites in Lower Aragon". En B. Frenzel (ed.): Evaluation of land surfaces cleared from forests by prehistoric man in Early Neolithic times and the time of migrating Germanic tribes. Paläoklimaforschung, Palaeoclimate Research 8: 129-138.

- (1992c): "Análisis polínicos". En M.L. Cerdeño y R. García (eds.): El Castro de la Coronilla. Ministerio de Cultura. Madrid: 141-144.

- (1993): "Estudios sobre la flora”. En A. Castiella (ed.): "De la Protohistoria navarra : la Edad del Hierro". Cuadernos de Arqueología de la Universidad de Navarra 1: 161-165.

- (1994): "Forest clearance and open land during the time of Roman Empire in Spain". Paläoklimaforschung, Palaeoclimate Research 10: 23-36.

- (1997) (coord.): El Paisaje Vegetal de la Comunidad de Madrid durante el Holoceno Final. Serie Arqueología, Paleontología y Etnografía, Monográfico 5. Madrid.

- (2000): "La reconstrucción del clima a través de la Palinología". En J.C. García Codrón (coord.): La reconstrucción del clima de época preindustrial: V Reunión nacional de Climatología (Santander 1998): 123-147. Santander.

LÓPez García, P. y ArnanZ, A.M. (1994): “Análisis polínico del yacimiento de El Ventorro (Madrid)". Estudios de Prehistoria y Arqueología Madrileñas 9: 57-58.

López García, P.; Arnanz, A.M.; UzQuiano, P. y LóPez SÁEz, J.A. (1997a): "Los elementos antrópicos en los análisis arqueobotánicos como indicadores de los usos del suelo". En J.M. García Ruíz, P. López García, (eds.): Acción humana y desertificación en ambientes mediterráneos. Instituto Pirenaico de Ecología. Zaragoza: 41-59.

LóPEZ, P.; CHERnYKh, E.N. y LóPEZ SÁEZ, J.A.(2001): “Palynological analysis at the Gorny site (Kargaly Region): The Earliest Metallurgical Centre in Northern Eurasia (Russia)". En D.K. Goodman and R.T. Clarke (ed.): Proceedings of the IX International Palynological Congress, Houston, Texas, EE.UU.1996. American Association of Stratigraphic Palynologists Foundation, Houston, Texas, EE.UU.: 347-355.

López García, P.; Díaz Fernández, P.M. y LóPez Sáez, J.A. (1992): “Análisis polínicos en la cuenca del río Deo". Revue de Paléobiologie 11: 205-211.

López García, P. y López SÁez, J.A. (1993): "Estudio polínico de cuatro túmulos megalíticos en la cuenca del río Ladra (Lugo, Galicia)". Trabajos de Prehistoria 50: 235-247.

- (1994a): "El paisaje andaluz en la Prehistoria: datos pa-

T. P., 59, n. ${ }^{\circ} 1,2002$ 
leopalinológicos". Revista Española de Micropaleontología 26: 49-59.

- (1994b): "Comparison of peats and archaeological samples in the Andalusian region, Spain". American Association of Stratigraphic Palynologists Contributions Series 29: 127-139.

- (1994c): "Contribución al conocimiento de la historia de la vegetación de Huesca: análisis palinológico del yacimiento de la Cueva del Moro (Olvena)". Boletín Geológico y Minero 105: 427-435.

- (1994d): "Estudio palinológico de los sedimentos arqueológicos del yacimiento del Llanete de los Moros (Córdoba)". Trabajos de Prehistoria 51: 179-186.

- (1996a): "La Cueva del Moro de Olvena (Huesca): La vegetación”. Bolskan 13: 143-146.

- (1996b): "Análisis paleopalinológico del yacimiento de 'Els Secans': dinámica de la vegetación durante el Cuaternario". Ãl-Qânnis 6: 84-89.

- (1996c): "El paisaje vegetal del yacimiento 'Montón de tierra', Griegos (Teruel)". Kalathos 11-12: 165-176.

- (1996d): "Degradación antropogénica de la vegetación de Sierra Mágina (Jaén) durante el Holoceno reciente". Nova Acta Científica Compostelana (Bioloxía) 6: 11-20.

- (1997): "Contribución al conocimiento de la historia de la vegetación de la provincia de Sevilla: análisis polínico del yacimiento arqueológico de 'Los Molares"'. Mediterranea. Serie de Estudios Biológicos 16: 19-22.

- (1998): "Análisis palinológico". En M. Molinos, T. Chapa, A. Ruiz, J. Pereira, C. Rísquez, C. Madrigal, A. Esteban, V. Mayoral, y M. Llorente (eds.): El santuario heróico de 'El Pajarillo' (Huelma, Jaén). Jaén: 111 119.

- (1999): "Contribución al conocimiento del paisaje Holoceno de la provincia de Soria: análisis palinológico en el yacimiento arqueológico de Parpantique". Acta Geologica Hispanica 31 (4): 77-84.

- (2000a): "Análisis polínico del Dolmen de Azután”. Revista Española de Micropaleontología 32 (1): 107-113.

- (2000b): "Le paysage et la phase Épipaléolithique-Mésolithique dans les Pré-Pyrénées Aragonaises et le Bassin Moyen de l'Èbre à partir de l'analyse palynologique". En Les derniers chasseurs-cueilleurs d'Europe occidentale (13 000 - 5500 av. J.-C.). Actes du Colloque international de Besançon, octobre 1998. Presses Universitaires Franc-Comtoises, Collection Annales Littéraires, 699; Série "Environnement, societés et archéologie". Besançon: 59-69.

López García, P.; López Sáez, J.A.; Aira, M.J. y SaA, P. (1993): “Análisis paleobotánico de cinco yacimientos arqueológicos de la Sierra del Bocelo (La Coruña, Galicia)". Anales de la Asociación de Palinólogos de Lengua Española 6: 59-73.

López García, P.; López SÁEZ, J.A. y Sánchez VillapadierNA, J.J. (1991): "Análisis polínico del yacimiento de Botiquería (Mazaleón, Teruel)". Trabajos de Prehistoria 48: 395-403.
López García, P.; LóPez Sáez, J.A. y UzQuiano, P. (1996a): "Paleoambiente y hábitat en las Marismas de Cantabria en los inicios del Holoceno: el caso del Abrigo de la Peña del Perro". En P. Ramil, C. Fernández y M. Rodríguez (coords.): Biogeografía Pleistocena-Holocena de la Península Ibérica. Consellería de Cultura, Xunta de Galicia, Santiago de Compostela: 333-348.

López García, P. y Molero, G. (1984): “Análisis de los restos vegetales, faunísticos y polínicos del yacimiento de La Sarsa (Bocairente, Valencia)". Trabajos de Prehistoria 41: 305-312.

López García, P.; UzQuiano, P.; López Sáez, J.A. y Gómez, C. (1996b): "Primeros datos sobre la paleovegetación cuaternaria de la Gran Estepa Euroasiática (Los Urales, Rusia)”. En B. Ruiz Zapata (eds.): Estudios Palinológico. Universidad de Alcalá de Henares. Alcalá de Henares: 75-78.

López García, P.; Uzquiano, P. y Arnanz, A. (1997b): “La arqueobotánica como medio de aproximación al conocimiento del papel del hombre en el proceso de cambio en la vegetación de la cuenca mediterránea". En J.J. Ibáñez, B.L. Valero Garcés y C. Machado (eds.): El paisaje mediterráneo a través del espacio y del tiempo. Implicaciones en la desertificación. Geoforma Ediciones. Logroño: 363-377.

LÓPEZ SÁEZ, J.A. (1992): "Sobre la conservación de la Flora leñosa y el Medio Ambiente en el sur de la Sierra de Gredos (Valle del Tiétar, Avila)". Actas de Gredos 12: 115-124.

- (1993): "Las alisedas (Scrophulario-Alnetum glutinosae) del Valle del Tiétar (Sierra de Gredos, Avila): estado de conservación y presencia de especies relícticas del Terciario y Pliocuaternario". En F.J. Silva Pando y G. Vega Alonso (eds.): Congreso Forestal Español (IV). Pontevedra: 41-46.

- (1995a): "La conservación de los bosques ribereños del Valle del Tiétar (Avila) y la introducción de especies exóticas". En A.R.B.A. y CO.MA.DEN. (eds.): II Encuentros sobre propagación de especies autóctonas y restauración de la vegetación natural (Madrid 1991): 74-77.

- (1995b): "Algunos táxones interesantes de la flora autóctona del sur de Gredos (Valle del Tiétar, Avila)". En A.R.B.A. y CO.MA.DEN. (eds.): II Encuentros sobre propagación de especies autóctonas y restauración de la vegetación natural (Madrid 1991): 165-168.

López SÁEZ, J.A.; Díaz Fernández, P.M. y SÁNCHEZ ViLlaPADIERNA, J.J. (1991): "La Criptogamia: Ciencia complemento de la Arqueología". Trabajos de Prehistoria 48: 383-393.

López SÁEz, J.A. y López García, P. (1992): “Dinámica del paisaje: un modelo de interpretación a través de la Paleopalinología”. En J.F. Martín Duque (ed.): V Jornadas sobre el Paisaje: Transformaciones del Paisaje en áreas de influencia de grandes núcleos urbanos (Segovia 1992): 375-385. Segovia. 
- (1994): "Contribution of the palaeoecological knowledge of Quaternary in the Tietar Valley (Sierra de Gredos, Avila, Spain)". Revista Española de Micropaleontología, 26: 61-66.

- (1999): "Rasgos paleoambientales de la transición Tardiglaciar-Holoceno (16-7.5 Ka BP) en el Mediterráneo ibérico, de Levante a Andalucía”. En Geoarqueologia i Quaternari litoral. Memorial M.P. Fumanal. Departament de Geografia, Universitat de València, Valencia: $139-152$.

López Sáez, J.A.; López García, P.; Gómez Ferreras, C. y Gil Hernández, P. (1996): “Acerca del origen del castaño (Castanea sativa) en el Valle del Tiétar (Sierra de Gredos, Avila)". En B. Ruiz Zapata (eds.): Estudios Palinológicos. Alcalá de Henares (Madrid): 79-82.

López Sáez, J.A.; López García, P. y Macías Rosado, R. (1991): "Análisis polínico del yacimiento arqueológico de El Raso de Candeleda (Avila)". Actas de Gredos 11: 39-44.

- (1997): “Acción antrópica y reconstrucción de la vegetación durante el Holoceno reciente en el valle del Tiétar, Sierra de Gredos (Avila)". Cuaternario y Geomorfología 11: 43-54.

LÓPEZ SÁEZ, J.A.; Martín SÁNCheZ, M. y LóPez GarCía, P. (1999): "Evolución del paisaje de Lanzahíta (Valle del Tiétar, Ávila) durante el Holoceno reciente: Una interpretación palinológica". Trasierra 4: 81-86.

LóPEz SÁEz, J.A.; TeXIER, P.J. y Bui-Thi-MAI (1998b): "Paléoenvironnement durant le Pléistocene Supérieur en Vaucluse: analyse palynologique des couches inférieures de l'Abri de La Combette (Bonnieux, Vaucluse, France)". Trabajos de Prehistoria 55 (2): 151-162.

López Sáez, J.A.; Van Geel, B.; Farbos-TeXier, S. y Diot, M.F. (1998a): "Remarques paléoécologiques à propos de quelques palynomorphes non-polliniques provenant de sédiments quaternaires en France". Revue de Paléobiologie 17 (2): 445-459.

Macías Rosado, R. y López García, P. (1999): "Análisis palinológico de los sedimentos del yacimiento de Melque (Toledo)". Archivo Español de Arqueología 72: 227-230.

Macías Rosado, R.; López García, P. y López Sáez, J.A.
(1998): "Análisis palinológicos en el sureste madrileño: yacimientos arqueológicos y depósitos naturales". Botánica Macaronésica 23: 241-253.

Martín SÁnchez, M.; López García, P. y López Sáez, J.A. (1999): “Aproximación palinológica a la evolución de la vegetación del Caúcaso y del nivel del mar del Mar Caspio durante el Holoceno reciente". Temas Geológico-Mineros ITGE 26: 269-273.

Martínez Atienza, F. (1999): "Bibliografía (1945-1998) paleopolínica del Holoceno Ibérico". Boletín de la Real Sociedad Española de Historia Natural (Sección Biología) 95: 5-30

Picazo, J., Yll, R.; Ros, M.T.; De la Torre, M.A.; SerraNO, I.; López García, P. y Blasco, M.F. (1997): “Subsistencia y medio ambiente durante la Edad del Bronce en el Sur del Sistema Ibérico Turolense". Teruel 85 (II): 9-48.

SÁEnz LaÍn, C. y Gutiérrez Bustillo, M. (1991): "Bibliografía palinológica española (1932-1988)". Lazaroa 12: 69-119.

SALAS, L. (1995): "Los estudios polínicos en España, utilizados en la reconstrucción climática de los últimos 10.000 años". Caderno Laboratorio Xeolóxico de Laxe 20: 67-98.

Targarona, J.; Alonso, B.; Cacho, I.; Canals, M. y López SÁEz, J.A. (1996): "Climatic changes during the Late Quaternary in southeast Spain: implications for the establishment of the Murciano-Almeriense bioprovince”. En B. Ruiz Zapata (ed.): Estudios Palinológicos. Universidad de Alcalá de Henares, Alcalá de Henares: 119-123.

Utrilla Miranda, P.; López García, P. y Mazo Pérez, C. (1986): "Interpretación microespacial de una ocupación magdaleniense a través de análisis polínicos y huellas de uso". Arqueología Espacial 8: 41-60.

Vicent García, J.M.; Rodríguez Alcalde, A.L.; López Sáez, J.A.; Zavala Morencos, I. de; López García, P. y Martínez Navarrete, M.I. (2000): “Catástrofes ecológicas en la estepa? Arqueología del Paisaje en el complejo minero-metalúrgico de Kargaly (Región de Orenburg, Rusia)". Trabajos de Prehistoria 57 (1): 29 74.

T. P., 59, n. ${ }^{\circ} 1,2002$ 